Running header: Music interventions

Music Interventions for Children with Autism: Narrative Review of the Literature

Kate Simpson

Deb Keen

Faculty of Education, Australian Catholic University 


\begin{abstract}
It is widely reported that music can be beneficial to individuals with autism. This review was undertaken to determine the evidence base for the use of music as an intervention for children with autism. After searching relevant databases, 128 articles were identified of which 20 articles met the study's inclusion criteria. Composed songs and improvisational music therapy were the predominant music techniques used. There was somewhat limited evidence to support the use of music interventions under certain conditions to facilitate social, communicative and behavioural skills in young children with autism. The implications of these findings in terms of use of music interventions, issues related to generalization and maintenance, and future research are discussed.
\end{abstract}

Keywords Autism, music, intervention, review Email: deb.keen@acu.edu.au 


\section{Music Interventions for Children with Autism: Review of the Literature}

Music has been used by therapists to facilitate communicative behaviours and social engagement with individuals with autism (Alvin, 1978; Alvin \& Warwick, 1992). The nonverbal aspect of music is viewed as a means of engaging the child and the therapist/ others in musical-emotional communication (Alvin, 1978; Alvin \& Warwick, 1992; Malloch \& Trevarthen, 2009). Much has been written about the purported benefits of music for individuals with autism based on the preference, responsivity and predisposition this group display to music stimuli.

A number of studies have reported that individuals with autism demonstrate a preference for auditory stimuli over other stimuli when the auditory stimuli is presented in the form of music (Blackstock, 1978; Kolko, Anderson, \& Campbell, 1980); and these individuals will engage with the auditory stimuli for a longer duration than typically developing children matched on developmental age (Thaut, 1987). Thaut (1988) measured musical responsiveness in a group of children with autism using music improvisations and found no significant difference in rhythm, restriction and originality compared to chronologically age matched peers, supporting the in-tact nature of musical responsiveness in this group.

Findings from experimental studies suggest that musical perception may also be preserved or enhanced in individuals with autism. Superior identification and labelling of pitch (Applebaum, Egel, Koegel, \& Imhoff, 1979; Bonnel et al., 2003; Heaton, Hermelin, \& Pring, 1998); enhanced sensitivity for pitch direction (Heaton, 2005) and enhanced detection of changes in pitch contours (Heaton, Hudry, Ludlow, \& Hill, 2008) have been demonstrated by individuals with autism when compared to matched controls. On auditory discrimination tasks using music stimuli, Jones et al. (2009) found that adolescents with autism performed as well as age and IQ matched controls; however a subgroup of individuals with autism demonstrated enhanced performance on the frequency discrimination task. Musical processing appears to be intact at both local and global levels (Heaton, Williams, Cummins, \& Happé, 2007; Mottron, Peretz, \& 
Menard, 2000) with a bias towards local processing when absolute pitch is used for discrimination (Mottron et al., 2000). Although some individuals with autism may display a preference and/or aptitude for elements of music, the implications of these abilities in terms of the impact on the efficacy of interventions need to be investigated.

A number of reviews investigating the use of music with individuals with autism have previously been conducted (Accordino, Comer, \& Heller, 2007; Gold, Wigram, \& Elefant, 2006; Whipple, 2004). Accordino and associates provided a narrative account of articles they identified as using music therapy from 1973 - 2000. The review encompassed the use of music therapy with children, adolescents and adults with autism to address social, behavioural and communicative outcomes. The parameters of the review limited the results to studies that only used music therapy and excluded studies that involved music in conjunction with intervention components. Of the 20 articles identified in the review, less than half were experimental design studies. With the exception of one study, the experimental studies identified used Auditory Integration Training.

In a Cochrane Report by Gold et al. (2006), three studies were selected which compared the use of music to non music conditions with children under the age of 10 years. Although significant results for gestural and verbal communicative skills during a music condition were reported, the studies were limited to receptive music therapy conducted within highly structured environments, with small numbers of young children. Gold et al. found there was little assessment by the researchers of generalization or maintenance following cessation of the intervention.

Whipple (2004) conducted a meta-analysis of nine studies comparing music and no music situations with children and adolescents with autism. Articles that described the use of music in any form were included. Whipple reported that music was effective as an intervention with individuals with autism irrespective of the age of participants, type of intervention, treatment, 
methodology and profession of the music provider. However, Whipple's review relied heavily on unpublished manuscripts, with only three articles published in peer reviewed journals being included.

The recent National Standards Project conducted by the National Autism Center (2009) classified music therapy as an emerging evidenced-based practice useful in teaching individual skills or goals by initially targeting the skill through song or rhythmic cuing. The project reported on six studies that used a range of music techniques. The project did not provide narratives of the studies or comparisons of treatment techniques.

Given the classification of music therapy as an emerging evidenced-based practice and the limitations of previous reviews, the aim of this paper was to review current research literature to determine the effectiveness of music interventions, the music technique used and the contexts of the intervention.

\section{Method}

\section{Procedure}

A comprehensive search of three selected computer databases, ERIC, Proquest and PsycINFO, using the search criteria 'autis*' and 'music' for the period 1990 - April 2010 was conducted. Articles were included for the current analysis if they met the following criteria:

1. Articles were published in a peer reviewed journal.

2. Participants were children (0-18 years) with a diagnosis of autism. Studies involving other special education populations were excluded regardless of inclusion of individuals with autism.

3. Participants demonstrated no savant behaviour in the area of music. The current review has focused on experimental studies that used music as an intervention or as a means to facilitate intervention. The studies associated with musical savants have generally focused 
on understanding their musical talents rather than considering music as an intervention.

4. Included studies demonstrated experimental control by using a music intervention to address an identified behaviour (Kennedy, 2005). Case studies and AB designs that did not demonstrate experimental control were excluded.

Articles that met the selection criteria were also identified through the National Standards Project (National Autism Center, 2009), and the three review articles, Accordino et al. (2007), Gold et al. (2006), and Whipple (2004). A further analysis excluded studies using treatments classified by the National Research Council (2001) as lacking theoretical support to substantiate or dispute the technique i.e. Auditory Integration Therapy and Facilitated Communication.

\section{Results}

Of the 128 studies located, 20 met the inclusion criteria. A list of excluded studies is available from the authors on request. The included studies were then categorized according to the focus of intervention, based on the skills and behaviours addressed (see Table 1). The focus areas were communication, socialization, and behaviour.

[place Table 1 about here]

\section{Music Used as an Intervention for Communication}

In the area of communication, two studies used music in conjunction with alternative and augmentative communication systems to facilitate communication with young children, during individual sessions (Buday, 1995; Simpson \& Keen, 2010). Buday (1995) compared music to rhythm conditions to determine if one condition was more effective in facilitating memory for signed words. In the music condition the text was sung, while in the rhythm condition the words were spoken rhythmically and at the same speed as the song. The number of signs and spoken words correctly imitated was significantly higher, and participants were observed to be more attentive during the music condition. However the study did not report on generalization of the signs and words to other contexts and maintenance of the symbols and signs over time. Simpson 
and Keen (2010) used a song embedded into a Powerpoint presentation to teach 3 young children displaying low level verbal comprehension skills to receptively label visual graphic symbols. A multiple-baseline design was used across participants that measured the children's correct number of responses. The music condition facilitated the receptive skills for each of the participants and performance was maintained when assessed three weeks following the cessation of intervention. Results showed little generalization of these skills to other contexts. In both studies the music consisted of generic songs that were pre-recorded and used during intervention.

Corbett, Shickman, and Ferrer (2008) compared the use of the Tomatis method of music intervention with a placebo condition to determine what effect, if any the individual music intervention had on improving receptive and expressive communication in a group of eleven children, aged 3-7 years. The Tomatis method can be described as a sound therapy that provides auditory stimulation by attenuating low frequency and amplifying higher frequency sounds in order to focus listening on language frequencies (Corbett, Shickman, \& Ferrer, 2008). There was no significant difference in language measures across the participants attributed to the treatment condition.

Using improvisational music therapy, Edgerton (1994) reported that music therapy increased the musical and non musical communicative behaviours, for a group of 11 children aged 6-9 years, during individual sessions. The communicative behaviours were measured using The Checklist of Communicative Responses/Acts Score Sheet (CRASS) designed specifically for the study to measure subject's musical and non musical communicative responses and acts. The CRASS predominantly assessed musical categories (91 of the 107 items) and communicative responses (69 of the 107 items). The reversal condition, consisting of one session using composed music, reported a substantial decrease in communicative acts and responses for all participants. Generalization of communicative behaviours assessed by parents, teachers and speech therapists using The Behaviour Change Survey reported minimal changes in behaviours 
in other contexts. Maintenance following the conclusion of the 10 week period was not assessed. A limitation of the B-A-B design is the absence of the initial baseline pattern of behaviour (Kennedy, 2005). Although experimental control was demonstrated by a decline in all behaviours during the reverse condition and an increase during intervention, the findings would demonstrate more confidence if the reverse condition consisted of more than one session.

\section{Music Used as an Intervention for Socialization}

A number of the studies reviewed used music as an intervention to develop socialization skills in children with autism. With the exception of Katagiri (2009), these studies included engagement as a focus of the research. Katagiri conducted individual sessions to teach decoding and encoding of four emotions - happiness, sadness, anger and fear. A counterbalance treatmentorder of four different instructional conditions: no contact control; contact control where verbal instructions accompanied visuals; background music utilizing pre-recorded improvised music designed to reflect the emotion and verbal instructions; and song texts using specifically composed songs about the emotion; was used. Participants demonstrated an increase in decoding skills in all conditions, with the background music condition being most effective. Improvements in decoding skills were higher than changes in encoding abilities. The nature of the counterbalanced situation resulted in participants improving decoding skills during the no contact control by a process of elimination. The researchers did not assess whether the skills achieved during intervention generalized to life situations and whether the individuals maintained the skills.

Two studies focusing on social engagement, compared music contexts to play contexts (Kern \& Aldridge, 2006; Kim, Wigram, \& Gold, 2008). Kern and Aldridge (2006) embedded music into an outdoor play context and compared the baseline conditions of no directed music activities to teacher and peer mediated music intervention. Individually composed songs, sung by teachers and peers resulted in an increase in positive peer interactions that was more apparent in 
the teacher initiated sessions. The authors acknowledged the lack of generalization and maintenance strategies in the study. This study provides some support for individualized interventions based on music therapy embedded into outdoor play routines and the involvement of peers to participate and model target behaviour. Classroom teachers and peers demonstrated the ability to learn and implement the program, with the teachers' accuracy of implementing the intervention being reported as high.

In the program research conducted by Kim et al. (2008), improvisational music therapy was compared to play sessions to determine the more effective condition for developing joint attention and positive emotional communication during individual sessions. The music condition was significantly more effective than the play sessions in increasing responses to joint attention and initiating low level joint attention acts i.e. eye contact while little change was observed in initiating high level joint attention behaviours, like pointing and showing. Children engaged in longer periods of turn-taking during music situation. Comparing the same conditions, Kim, Wigram, and Gold (2009) rated the frequency of 'joy', 'emotional synchronicity', and 'initiation of engagement' more higher and longer durations of 'joy' and 'emotional synchronicty' during the music compared to play sessions. The frequency and duration of these behaviours was higher during the undirected session versus the directed session of music. Compliance response was more frequent during the music session, while no response was more frequent during the play session. These studies provide some evidence that music may facilitate a number of joint attention and affective behaviours; however generalization to other settings was not investigated.

The use of music has also been incorporated into a playful context. Wimpory, Chadwick, and Nash (1995) used musical interaction therapy with a 3-year-old and her mother. The intervention led to improvements in the area of social engagement evidenced by increased use of eye contact and initiations of interactive involvement. These skills were maintained when assessed at followup 20 months later. 
Stephens (2008) conducted a study using a music context during individual sessions to create a social routine for the purpose of engaging the participants in reciprocal imitation behaviours. The sessions used music to establish a repetitive imitating routine prior to children being provided the opportunity to imitate adults modelled action and word pairs. The music was used to define the play routines and to distinguish between the researcher's behaviour. The focus was on the participants' willingness to engage spontaneously with the adult. Spontaneous action and word imitation was demonstrated by 3 of the 4 participants during the social interaction, but was only maintained during the probe situation for 2 of the 4 participants.

Kern, Wolery, and Aldridge (2007) embedded music into morning routines to increase independent functioning of two children aged 3 years. Individualised lyrics were composed to a common melody for each participant. Teaching staff were trained and provided with the song and song transcripts. Kern and associates reported the use of the song facilitated independent functioning and peer initiated interaction. The teachers reported the interventions were effective but they were challenged by structural elements of the song. Maintenance and generalization data were not reported.

\section{Music Used as an Intervention for Behaviour}

The predominant number of studies in this review focused on the use of music intervention to regulate behaviour. Two studies implemented music interventions in conjunction with social stories (Brownell, 2002; Pasiali, 2004). Social stories are short stories designed to aid in the social understandings of individuals with autism (Gray, 2000). In both studies, the music therapist used the social story format to create prescriptive songs addressing the individual's target behaviour. Brownell (2002) compared reading and singing versions of the social story using a counterbalanced treatment order and reported a significant reduction of the target challenging behaviour for all four participants in both conditions. Although the targeted behaviour was recorded less frequently in the singing condition, it only reached statistical 
significance for one participant. Pasiali (2004) introduced the prescriptive song protocol as an individual intervention conducted in the home environment. Two of the three children demonstrated a significant reduction in behaviour between the baseline and intervention. Although the third child did not display a significant reduction in behaviour, the child did demonstrate an increase in the more appropriate alternate behaviour described in the song. Pasiali observed the use of the naturalistic environment contributed to a number of unpredictable factors that were difficult to control and may have influenced performance including a possible bias by parents in scoring. The decrease in behaviour was maintained at follow-up for all participants however there was a lack of generalization which Pasiali attributed partially due to the specificality of the behaviours to the home environment.

Carnahan, Basham, and Musti-Rao (2009) and Carnahan, Musti-Rao, and Bailey (2009), explored the use of music within a group setting to determine the effect music had on levels of active engagement during an interactive book session. This was assessed by measuring attending behaviour. Carnahan and associates compared three conditions: read only; interactive story using two and three dimensional materials; and music added with text sung. A system of observations occurred where each child was observed during a 6 second interval. Results indicate levels of engagement increased for all children when interactive books were used with music. The intervention was implemented by the class teacher who was positive about the intervention and the ease of implementing it within the daily classroom schedule.

The remaining studies used music as auditory stimuli to regulate behaviour. Orr, Smith Myles, and Carlson (1998) reported that the use of specifically metered music reduced aberrant behaviour in an 11 year-old girl during situations she found moderately stressful. Gunter and Fox (1993) compared the use of music which was either non-contingent or contingent on task performance, for a 14 year-old boy during vocational training. For this student, the use of noncontingent music was more effective in reducing aberrant behaviour. Similar effects were 
reported by Rapp (2007). Rapp compared the contingent use of music played on a CD with music and toys; no music and toys; and no music, no toys. Levels of vocal stereotypy displayed by the participant, a 9 year-old boy with autism, were lowest in the contingent music condition, suggesting that auditory stimuli may be functionally matched to the product of vocal stereotypy. In another study assessing impacts of auditory stimuli on vocal stereotypy Lanovaz, Fletcher, and Rapp (2009) compared four conditions, two that matched the sensory product of stereotypy (music and musical toys), and two that provide no matched sensory product (toys that provided no auditory feedback and no toy situations). Their results indicated that for 2 of the three participants, vocal stereotypy was not specific to the matched sensory product.

Not all individuals with autism find listening to music a positive experience. Devlin, Healy, Leader and Reed (2008) observed an increase in challenging behaviour displayed by a six yearold when he was exposed to certain types of music. In their study they were able to increase the boy's tolerance for the music by using it as a differential negative reinforcement of other behaviour.

\section{Discussion}

The current study identified 20 experimental studies for review to evaluate the effectiveness of music as an intervention with individuals with autism. The included studies used music interventions to target communication, socialization and behavioural skills. This is consistent with Kaplan and Steele's (2005) findings that identified $80 \%$ of primary music therapy goals were recorded in the area of language/communication and behaviour/psychosocial. In the review, a number of study characteristics emerged that may potentially impact on the effectiveness of music interventions. The music techniques ranged from improvised sessions to highly structured techniques, highlighting the need to consider the impact of music techniques and specific components of these techniques on the dependent variables identified in each study. The review also drew attention to the importance of evaluating intervention context, (e.g. naturalistic and 
experimental settings), the interventionist's level of training, and participant characteristics as they related to the efficacy of the intervention.

\section{Music Technique}

Improvisational music therapy and the use of composed songs were the predominant forms of music interventions reported in the reviewed studies. In the improvisational music therapy interventions the music context evolved during the sessions, while in the composed song intervention the music and or lyrics were composed prior to the intervention and implemented using a recording or a sung presentation. Both techniques demonstrated some support for the use of music to facilitate target skills in the areas of communication and socialization, while two studies found composed songs to be effective in targeting particular behaviours. In a number of studies, composed songs were used in conjunction with other interventions which may have contributed to the effectiveness of this type of music intervention and highlights the need for improved research designs that isolate different components of the intervention to determine their relative contributions.

One study found that improvisational music therapy was more effective than set songs in facilitating change in targeted skills (Edgerton, 1994). However in this study, the individuals were exposed to proportionally more improvisational sessions than composed song sessions, making it unclear how the type rather than the length of intervention influenced the outcome. The improvisational technique allows the therapist to be responsive to the child and to facilitate active and receptive music elements, while the structured set songs rely on receptive music techniques and allow for little flexibility in the mode of presentation. From the results of the studies reviewed here, it is unclear whether these elements can impact on the effectiveness of the intervention. Two music techniques identified in the review, auditory stimuli and Tomatis method, demonstrated no link between the music intervention and changes in the individual's learning outcomes. 


\section{Context for Intervention}

Although a number of studies were embedded into the natural classroom environment or home situation the majority of the studies were conducted in experimental environments. The nature of a number of the interventions, particularly when music was used in conjunction with other strategies, displayed the potential for these strategies to be embedded within a naturalistic environment. However, Pasiali (2004) observed implementing the intervention in the naturalistic environment generated a number of external variables that were difficult to control and may have impacted on the experimental research. Although music interventions may demonstrate efficacy within the experimental environment to support inclusive and naturalistic education it will be important to demonstrate effectiveness in more naturalistic settings.

\section{Training}

There is little research on the core skills training required for interventionists to implement music interventions with children with autism. Improvisational music therapy, a technique used in a number of the reviewed studies, is an insight oriented therapy that was implemented by certified music therapists; while the composed song interventions were implemented by music therapists, class teachers, family members and peers. Music stimuli used in the composed song interventions ranged from songs specifically composed by the music therapist (Brownell, 2002; Kern and Aldridge, 2006; Kern et al., 2007; Pasiali, 2004), original lyrics composed by a music therapist using familiar melodies (Pasiali, 2004) and the use of conventional songs (Buday, 1995; Carnahan, Basham et al., 2009; Carnahan, Musti-Rao et al., 2009; Simpson \& Keen, 2010). One class teacher viewed using the conventional song as easy to implement in the group situation (Carnahan, Basham et al., 2009; Carnahan, Musti-Rao et al., 2009), while other teachers and parents experienced difficulties with the complexity of the composed songs (Kern \& Aldridge, 2006; Pasiali, 2004). Embedding a traditional song into a Powerpoint presentation (Simpson \& 
Keen, 2010) eliminated the need to provide musical training to the teacher and may be a useful area to pursue in future research.

\section{Participants}

One selection criteria for included studies in this review was children aged 0-18 years. The use of music interventions with individuals with autism predominantly focused on young children (3-11yrs), with only 2 of the 106 participants in the adolescent age range (12-18yrs). There is little published research involving participants aged 11-18 years making it difficult to determine whether music interventions may be efficacious for this age group.

\section{Generalization and Maintenance}

Questions of generalization and maintenance of target behaviours acquired through music interventions remain, as few of the studies included these measures in their design. When studies did include generalization measures, there was little generalization of skills to other contexts (Edgerton, 1994; Pasiali, 2004; Simpson \& Keen, 2010), raising questions about the social validity of these interventions in the lives of children with autism. Maintenance of the skills following the cessation of intervention was only reported in two studies (Pasiali, 2004; Simpson \& Keen, 2010). As individuals with autism experience particular difficulty in generalizing skills to contexts other than the training context and comprehension is often only in highly familiar contexts (Lovaas, Koegel, Simmons, \& Long, 1973), this creates questions as to the individual's level of comprehension during intervention or whether they are responding to the contexts and cues provided during the intervention.

\section{Future Directions}

Over the last 20 years there has been an increase in the number of experimental studies investigating the use of music as an intervention with children with autism. This has been prompted by a lack of evidence in this area and the demand for investigating and identifying effective interventions to be used with children with autism. In a review of educational and 
behavioural treatments that target the core characteristics of autism the use of music has been identified as an emerging practice, however additional studies are required before treatment effectiveness can be determined (National Autism Centre, 2009).

The studies reviewed provide some initial although limited evidence about the efficacy of music interventions with children with autism. These studies were generally conducted with small numbers using a range of techniques and applications. A number of them incorporated the use of music as one component of a multicomponent intervention. There is a need for comparative studies to identify the effectiveness of the music component of these interventions.

It appears that some individuals with autism may respond to elements of music and more research is required into the efficacy of specific applications of music stimuli. Given the support for music to facilitate engagement it would be informative to observe if this intervention context then facilitates learning. There is preliminary evidence that children with autism may benefit from music interventions within naturalistic settings and further investigation into these types of interventions and the training required to implement them is required. Although music intervention has been used to facilitate social, behaviour and communication skills, further research is required to establish the contribution of these interventions to the maintenance and generalization of these skills. 


\section{References}

Accordino, R., Comer, R., \& Heller, W. B. (2007). Searching for music's potential: A critical examination of research on music therapy with individuals with autism. Research in Autism Spectrum Disorders, $1,101-115$.

Alvin, J. (1978). Music therapy for the autistic child. Oxford: Oxford University Press.

Alvin, J., \& Warwick, A. (1992). Music therapy for the autistic child (2nd ed.). Oxford: Oxford University Press.

Applebaum, E., Egel, A. L., Koegel, R. L., \& Imhoff, B. (1979). Measuring musical abilities of autistic children. Journal of Autism and Developmental Disorders, 9, 279-285.

Blackstock, E. G. (1978). Cerebral asymmetry and the development of early infantile autism. Journal of Autism and Childhood Schizophrenia, 8, 339-353.

Bonnel, A., Mottron, L., Peretz, I., Trudel, M., Gallum, E., \& Bonnel, A. (2003). Enhanced pitch sensitivity in individuals with autism: A signal detection analysis. Journal of Cognitive Neuroscience, 15, 206-235.

Brownell, M. D. (2002). Musically adapted social stories to modify behaviours in students with autism: Four case studies. Journal of Music Therapy, 39, 117-144.

Buday, E. M. (1995). The effects of signed and spoken words taught with music on sign and speech imitation by children with autism. Journal of Music Therapy, 32, 189-202.

Carnahan, C., Basham, J., \& Musti-Rao, S. (2009). A low-technology strategy for increasing engagement of students with autism and significant learning needs. Exceptionality, 17, 76-87.

Carnahan, C., Musti-Rao, S., \& Bailey, J. (2009). Promoting active engagement in small group learning experiences for students with autism and significant learning needs. Education \& Treatment of Children, 32, 37-62.

Corbett, B. A., Shickman, K., \& Ferrer, E. (2008). Brief Report: The effects of Tomatis Sound in children with autism. Journal of Autism and Developmental Disorders, 38, 562-566. 
Devlin, S., Healy, O., Leader, G., \& Reed, P. (2008). The analysis and treatment of problem behaviour evoked by auditory stimulation. Research in Autism Spectrum Disorders, 2, 671-680.

Edgerton, C. L. (1994). The effect of improvisational music therapy on the communicative behaviours of autistic children. Journal of Music Therapy, 31, 31-62.

Gold, C., Wigram, T., \& Elefant, C. (2006). Music therapy for autistic spectrum disorder. Cochrane Database of Systematic Reviews, Issue 2. Art. No.: CD004381. DOI: 10.1002/14651858.CD004381.pub2.

Gray, C. (2000). The new social story book: Illustrated edition. Arlington: Future Horizons.

Gunter, P., \& Fox, J. (1993). A case study of the reduction of aberrant, repetitive responses of an adolescent with autism. Education and Treatment of Children, 16, 187-197.

Heaton, P. (2005). Interval and contour processing in autism. Journal of Autism and Developmental Disorders, 35, 787-793.

Heaton, P., Hermelin, B., \& Pring, L. (1998). Autism and pitch processing: A precursor for savant musical ability? Music Perception, 15, 291-305.

Heaton, P., Hudry, K., Ludlow, A., \& Hill, E. (2008). Superior discrimination of speech pitch and its relationship to verbal ability in autism spectrum disorders. Cognitive Neuropsychology, 25, 771782.

Heaton, P., Williams, K., Cummins, O., \& Happé, F. (2007). Beyond perception: Musical representational processing in autism. Journal of Autism and Developmental Disorders, 37, 1355-1360.

Jones, C., Happé, F., Baird, G., Simonoff, E., Marsden, A., Tregay, J., et al. (2009). Auditory discrimination and auditory sensory behaviours in autism spectrum disorders. Neuropsychologia, 47, 28502858.

Kaplan, R. S., \& Steele, A. L. (2005). An analysis of music therapy program goals and outcomes for clients with diagnoses on the autism spectrum. Journal of Music Therapy, 42, 2-19.

Katagiri, J. (2009). The effect of background music and song texts on the emotional understanding of children with autism. Journal of Music Therapy, 46, 15-31. 
Kennedy, C. H. (2005). Single-case designs for educational research. Boston: Pearson Education.

Kern, P., \& Aldridge, D. (2006). Using embedded music therapy interventions to support outdoor play of young children with autism in an inclusive community-based child care program. Journal of Music Therapy, 43, 270-294.

Kern, P., Wolery, M., \& Aldridge, D. (2007). Use of songs to promote independence in morning greeting routines for young children with autism. Journal of Autism and Developmental Disorders, 37, $1264-1271$.

Kim, J., Wigram, T., \& Gold, C. (2008). The effects of improvisational music therapy on joint attention behaviours in autistic children: A randomized controlled study. Journal of Autism and Developmental Disorders, 38, 1758-1766.

Kim, J., Wigram, T., \& Gold, C. (2009). Emotional, motivational and interpersonal responsiveness of children with autism in improvisational music therapy. Autism, 13, 389-409.

Kolko, D. J., Anderson, L., \& Campbell, M. (1980). Sensory preference and overselective responding in autistic children. Journal of Autism and Developmental Disorders, 10, 259-271.

Lanovaz, M., Fletcher, S., \& Rapp, J. (2009). Identifying stimuli that alter immediate and subsequent levels of vocial stereotypy: A further analysis of functionally matched stimulation. Behaviour Modification, 33, 682-704.

Lovaas, O. I., Koegel, R. L., Simmons, J. Q., \& Long, J. (1973). Some generalization and follow-up measures on autistic children in behaviour therapy. Journal of Applied Behaviour Analysis, 6, 131-166.

Malloch, S., \& Trevarthen, C. (2009). Musicality: Communicating the vitality and interests of life. In S. Malloch \& C. Trevarthen (Eds.), Communicative musicality: Exploring the basis of human companionship (pp. 1-15). Oxford: Oxford University Press.

Mottron, L., Peretz, L., \& Menard, E. (2000). Local and global processing of music in high-functioning persons with autism: Beyond central coherence? Journal of Child Psychology and Psychiatry, 8 , 1057-1065. 
National Autism Center. (2009). National Standards Project: Addressing the need for evidence-based practice guidelines for autism spectrum disorder. Findings and conclusions. Retrieved from http://www.nationalautismcenter.org/pdf/NAC\%20Findings\%20\&\%20Conclusions.pdf

National Research Council. (2001). Educating children with autism. Washington, DC.: National Academy Press.

Orr, T., Myles, B. S., \& Carlson, J. K. (1998). The impact of rhythmic entrainment of a person with autism. Focus on Autism and other Developmental Disabilities, 13, 163-166.

Pasiali, V. (2004). The use of prescriptive therapeutic songs in a home-based environment to promote social skills acquisition by children with autism: Three case studies. Music Therapy Perspectives, 20, 11-20.

Rapp, J. (2007). Further evaluation of methods to identify matched stimulation. Journal of Applied Behaviour Analysis, 40, 73-88.

Simpson, K., \& Keen, D. (2010). Teaching young children with autism graphic symbols embedded within an interactive song. Journal of Developmental and Physical Disabilities, 20, 165-177.

Stephens, C. E. (2008). Spontaneous imitation by children with autism during a repetitive musical play routine. Autism, 12, 645-671.

Thaut, M. (1987). Visual versus auditory (musical) stimulus preferences in autistic children: A pilot study. Journal of Autism and Developmental Disorders, 17, 425-432.

Thaut, M. (1988). Measuring musical responsiveness in autistic children: A comparative analysis of improvised musical tone sequences of autistic, normal, and mentally retarded individuals. Journal of Autism and Developmental Disorders, 18, 561-571.

Whipple, J. (2004). Music in intervention for children and adolescents with autism: A meta-analysis. Journal of Music Therapy, 41, 90-106.

Wimpory, D., Chadwick, P., \& Nash, S. (1995). Brief report: Musical interaction therapy for children with autism: An evaluative case study with two-year follow-up. Journal of Autism and Development Disorders, 25, 541-552. 


\section{Author Note}

This review is part of the first authors $\mathrm{PhD}$ study and is supported by a PhD scholarship - The Australian Catholic University Postgraduate Award. Address correspondence to Professor Deb Keen, Faculty of Education, Australian Catholic University, Queensland Australia 4014. deb.keen@acu.edu.au 
Table 1

Music studies

\begin{tabular}{|c|c|c|c|c|c|}
\hline Author & \multicolumn{2}{|r|}{ Study } & \multicolumn{2}{|c|}{ Participants } & Focus of Intervention \\
\hline Buday & 1995 & Within subject design & 10 & $4-9 y$ & Communication \\
\hline Carnahan, Musti-Rao \& Bailey & 2009 & A-B-C-A-C reversal & 6 & $6-11 y$ & Behaviour \\
\hline Corbett, Shickman, \& Ferrer & 2008 & $\begin{array}{l}\text { Double blind, placebo-controlled, } \\
\text { crossover }\end{array}$ & 11 & $3-7 y$ & Communication \\
\hline Gunter \& Fox & 1993 & $\begin{array}{l}\text { Multiple baseline design combined } \\
\text { with a withdrawal of treatment }\end{array}$ & 1 & $14 y$ & Behaviour \\
\hline Katagiri & 2009 & Counterbalance treatment design & 12 & $9-15 y$ & Socialization \\
\hline Kern \& Aldridge & 2006 & $\begin{array}{l}\text { Multiple baseline design across } \\
\text { participants. }\end{array}$ & 4 & $3-5 y$ & Socialization \\
\hline
\end{tabular}


Table 1 (continued)

\begin{tabular}{|c|c|c|c|c|c|}
\hline Author & \multicolumn{2}{|r|}{ Study } & \multicolumn{2}{|c|}{ Participants } & Focus of Intervention \\
\hline & & 2. $\mathrm{A}-\mathrm{B}-\mathrm{A}-\mathrm{B}$ & & & \\
\hline & & comparison in two different & & & \\
\hline & & conditions. & & & \\
\hline Kim, Wigram \& Gold & 2009 & Random control, single subject & 10 & $3-5 y$ & Socialization \\
\hline Orr, Myles, \& Carlson & 1998 & A-B-A-B & 1 & $11 \mathrm{y}$ & Behaviour \\
\hline Pasiali & 2004 & A-B-A-B reversal & 3 & $7-9 y$ & Behaviour \\
\hline Rapp & 2007 & Reverse design & 1 & $9 y$ & Behaviour \\
\hline Simpson \& Keen & 2010 & Multi-baseline design across participants & 3 & $3-4 y$ & Communication \\
\hline
\end{tabular}


Music Interventions 24

Table 1 (continued)

\begin{tabular}{|c|c|c|c|c|c|}
\hline Author & \multicolumn{2}{|r|}{ Study } & \multicolumn{2}{|c|}{ Participants } & Focus of Intervention \\
\hline Stephens & 2008 & $\begin{array}{l}\text { Multiprobe design across behaviour tiers } \\
\text { (3 participants) }\end{array}$ & 4 & $5-9 y$ & Socialization \\
\hline Wimpory, Chadwick \& Nash & 1995 & A-B-C design & 1 & $3.3 y$ & Socialization \\
\hline
\end{tabular}

